TRANSACTIONS OF THE

AMERICAN MATHEMATICAL SOCIETY

Volume 356, Number 8 , Pages 3069-3076

S 0002-9947(03)03490-

Article electronically published on November 4, 2003

\title{
SZEGÖ KERNELS AND FINITE GROUP ACTIONS
}

\author{
ROBERTO PAOLETTI
}

\begin{abstract}
In the context of almost complex quantization, a natural generalization of algebro-geometric linear series on a compact symplectic manifold has been proposed. Here we suppose given a compatible action of a finite group and consider the linear subseries associated to the irreducible representations of $G$, give conditions under which these are base-point-free and study properties of the associated projective morphisms. The results obtained are new even in the complex projective case.
\end{abstract}

\section{INTRODUCTION}

Let $(M, \omega)$ be a compact symplectic manifold of dimension $2 n$, such that $[\omega] \epsilon$ $H^{2}(M, \mathbb{Z})$. Fix $J \in \mathcal{J}(M, \omega)$ (the contractible space of all almost complex structures on $M$ compatible with $\omega$ ), and let $h$ and $g=\mathcal{R}(h)$ be the induced hermitian and riemannian structures. There exist an hermitian line bundle $(A, h)$ on $M$ and a unitary covariant derivative $\nabla_{A}$ on $A$, such that $-2 \pi i \omega$ is the curvature of $\nabla_{A}$.

In this set-up, the usual $\bar{\partial}$-complex from complex geometry can be replaced by a complex of pseudodifferential operators enjoying similar symbolic properties BdM], BdMG]; building on this foundational result, a theory of almost complex quantization has been developed and studied by several authors.

Namely, one can define spaces of quantized sections

$$
H\left(M, A^{\otimes k}\right) \subseteq \mathcal{C}^{\infty}\left(M, A^{\otimes k}\right),
$$

as the kernel of the first operator in the complex. A related approach is in terms of the asymptotic spectral properties of a suitable renormalized laplacian [GU], BU1].

These linear series determine projective embeddings of $M$ enjoying the same metric and symplectic asymptotic properties as in the integrable projective case BU2, [Z, SZ2, [T]. In the integrable case the theory reduces to the usual classical constructions of complex algebraic geometry.

Suppose now that $G$ is a finite group with a symplectic action

$$
\nu: G \times M \rightarrow M,
$$

so that $J$ may be chosen $G$-invariant. Then $\nu$ preserves $g$ and $h$. Assume also that $\nu$ lifts to a linear action $\tilde{\nu}: G \times A \rightarrow A$, and that $\tilde{\nu}$ preserves $h_{A}$ and $\nabla_{A}$. Then $\tilde{\nu}$ preserves each of the spaces $H\left(M, A^{\otimes N}\right)$. Let

$$
\rho_{i}: G \rightarrow \mathrm{GL}\left(V_{i}\right) \quad(1 \leq i \leq c)
$$

Received by the editors January 10, 2003.

2000 Mathematics Subject Classification. Primary 14A10, 53D50, 57S17. 
be the irreducible representations of $G$; we shall assume that $i=1$ corresponds to the trivial one-dimensional representation. For each $N$, we have a $G$-equivariant decomposition

$$
H\left(M, A^{\otimes N}\right)=\bigoplus_{i=1}^{c} H\left(M, A^{\otimes N}\right)_{i}
$$

where $H\left(M, A^{\otimes N}\right)_{i}$ consists of a direct sum of copies of $V_{i}$. It is natural to ask whether the linear series $\left|H\left(M, A^{\otimes N}\right)_{i}\right|$ are base-point-free and, if so, what about their asymptotic properties? In this note, we apply arguments from [BU2] and [Z], SZ2 to these questions.

If $x \in M$, let $G_{x}=\{g \in G: g \cdot x=x\}$ be its stabiliser. Let $\chi_{i}: G \rightarrow \mathbb{C}$ be the character of the $i$-th irreducible representation. Let $A_{x}$ be the fibre of $A$ over $x \in M$. Clearly, $G_{x}$ acts on $A_{x}$ and thus we have a unitary character $\alpha_{x}: G_{x} \rightarrow S^{1} \subset \mathbb{C}^{*}$. Let

$$
\gamma_{i, N}(x):=\left(\alpha_{x}^{N}, \chi_{i}\right)_{G_{x}}=\sum_{g \in G_{x}} \alpha_{x}(g)^{N} \cdot \bar{\chi}_{i}(g) \quad(x \in M, 1 \leq i \leq c, N \in \mathbb{N}),
$$

$(,)_{G_{x}}$ denoting the $L^{2}$-product with respect to the counting measure on $G_{x}$. Note that $\gamma_{i, N}=\gamma_{i, N+|G|}$ for every $i$ and $N$, where $|G|$ denotes the order of $G$. Set

$$
B_{i, N}:=\left\{x \in M: \gamma_{i, N}(x)=0\right\}=B_{i, N+|G|} \quad(1 \leq i \leq c) .
$$

Clearly, $x \in B_{i, N}$ implies $G_{x} \neq\{e\}$.

Our first goal is to determine the base locus of the spaces of sections $H\left(M, A^{\otimes k}\right)_{i}$ for $k \gg 0$. In algebro-geometric terminology, the base locus of a vector subspace $W \subseteq \mathcal{C}^{\infty}\left(M, A^{\otimes N}\right)$ is

$$
\operatorname{Bs}(|W|):=\{x \in M: s(x)=0 \forall s \in W\} .
$$

To begin with, we shall prove:

Theorem 1.1. Suppose $1 \leq i \leq c, 0 \leq r \leq|G|-1, x \in M$ and $\gamma_{i, r}(x) \neq 0$. Then for $N \gg 0, N \equiv r(\bmod |G|)$ there exists a section $s \in H\left(M, A^{\otimes N}\right)_{i}$ such that $s(x) \neq 0$.

This has a number of consequences:

Corollary 1.1. Suppose that the action of $G$ on $M$ is effective. Then

$$
\operatorname{dim}\left(H\left(M, A^{\otimes k}\right)_{i}\right)>0
$$

for every $i=1, \ldots, c$ and every $k \gg 0$.

In fact, it is proved in $[\mathrm{P}]$ that under the same hypothesis

$$
\operatorname{dim}\left(H\left(M, A^{\otimes k}\right)_{i}\right)=\frac{\operatorname{dim}\left(V_{i}\right)^{2}}{|G|} \cdot \frac{k^{n}}{n !} \cdot c_{1}(A)^{n}+o\left(k^{n}\right) .
$$

Proposition 1.1. Suppose $1 \leq i \leq c, 0 \leq r \leq|G|-1$, and $\gamma_{i, r}(x) \neq 0$ for every $x \in M$. Then $H\left(M, A^{\otimes k}\right)_{i}$ globally generates $A^{\otimes k}$ if $k \gg 0$ and $k \equiv r(\bmod |G|)$, that is, for every $x \in M$ there is $s \in H\left(M, A^{\otimes k}\right)_{i}$ such that $s(x) \neq 0$.

Corollary 1.2. If $k \gg 0$ and $i=1, \ldots, c$, the subspace of $G$-invariant sections

$$
H\left(M, A^{\otimes k|G|}\right)^{G} \subseteq H\left(M, A^{\otimes k|G|}\right)
$$

globally generates $A^{\otimes k|G|}$. 
Corollary 1.3. If $M$ is a complex projective manifold and $A$ is ample, for every $i=1, \ldots, c$ and $r=0, \ldots,|G|-1$ the base loci $\operatorname{Bs}\left(\left|H^{0}\left(M, A^{\otimes(r+k|G|)}\right)_{i}\right|\right)$ stabilize for $k \gg 0$. Furthermore, for every $k \gg 0$,

$$
\operatorname{Bs}\left(\left|H^{0}\left(M, A^{\otimes(r+k|G|)}\right)_{i}\right|\right) \subseteq B_{i, r} .
$$

In the reverse direction, it is easily seen that if $G_{x}=G$ and there exists $s \in$ $\mathcal{C}^{\infty}\left(M, A^{\otimes N}\right)_{i}$ with $s(x) \neq 0$, then

$$
\left(\alpha_{x}^{N}, \chi_{i}\right)_{G} \neq 0 .
$$

Therefore,

Corollary 1.4. In the hypothesis of Corollary 1.3, suppose in addition that either $G_{x}=\{e\}$ or $G_{x}=G$ for every $x \in G$. Then

$$
\operatorname{Bs}\left(\left|H\left(M, A^{\otimes N}\right)_{i}\right|\right)=B_{i, N}
$$

for $i=1, \ldots, c$ and $N \gg 0$.

In the almost complex case, for any $i=1, \ldots, c$ and $r=0, \ldots,|G|-1$ we may still define the $(i, r)$-th equivariant asymptotic base locus of $A$ as

$$
\begin{array}{r}
\operatorname{Bs}(A, i, r)_{\infty}=:\{x \in M: \forall s>0 \exists k>s, k \equiv r(\bmod |G|) \\
\text { such that } \left.x \in \operatorname{Bs}\left(\left|H\left(M, A^{\otimes k}\right)_{i}\right|\right)\right\} .
\end{array}
$$

The general case (symplectic, almost complex) of Corollary [1.3] is then

Corollary 1.5. In the above situation,

$$
\operatorname{Bs}(A, i, r)_{\infty} \subseteq B_{i, r} .
$$

If furthermore $K \subset M$ is any compact subset with $K \cap B_{i, r}=\emptyset$, then

$$
K \cap \operatorname{Bs}\left(\left|H\left(M, A^{\otimes k}\right)_{i}\right|\right)=\emptyset
$$

for all $k \gg 0$ with $k \equiv r(\bmod |G|)$.

Next, if Bs $\left(\left|H\left(M, A^{\otimes N}\right)_{i}\right|\right)=\emptyset$, there are associated projective morphisms

$$
\Phi_{i, r+k|G|}: M \rightarrow \mathbb{P}\left(H\left(M, A^{\otimes(r+k|G|)}\right)_{i}^{*}\right),
$$

and we now consider their asymptotic properties as $k \rightarrow+\infty$.

Theorem 1.2. Suppose $\operatorname{Bs}\left(\left|H\left(M, A^{\otimes N}\right)_{i}\right|\right)=\emptyset$ for some $1 \leq i \leq c$ and $0 \leq r \leq$ $|G|-1$. Let $U \subseteq M$ be the open subset of $M$ where the order $\left|G_{x}\right|$ is locally constant. Suppose $U^{\prime} \subset U$ is open with $\overline{U^{\prime}} \subset U$. Then $\Phi_{i, r+k|G|}$ is an immersion on $U^{\prime}$ for $k \gg 0$.

Corollary 1.6. $\left|H\left(M, A^{\otimes N}\right)^{G}\right|$ is base-point-free and $\Phi_{1, N}$ is an immersion on compact subsets of $U$ if $N \gg 0$ and $\sum_{g \in G_{x}} \alpha_{x}(g)^{N} \neq 0$ for every $x \in G$.

In general $\Phi_{i, N}$ is not injective; for example it is constant on every orbit for any $G$ if $i$ corresponds to the trivial representation, or for any $i$ if $G$ is abelian. We may still ask, however, if in these cases points in different orbits have different images under $\Phi_{i, N}$.

Let $d_{G}: M \times M \rightarrow \mathbb{R}$ be the orbit distance:

$$
d_{G}(x, y):=\min \{d(g x, y): g \in G\} \quad(x, y \in M) .
$$

Clearly, $d_{G}(x, y)>0$ if and only if $x \notin G \cdot y$. 
Proposition 1.2. Assume that either $G$ is abelian, or $G$ is arbitrary and $i=1$. Let $U \subseteq M$ be as in Theorem $1.1, N \in \mathbb{N}$ and suppose that $\operatorname{Bs}\left(\left|H\left(M, A^{\otimes N}\right)_{i}\right|\right)=\emptyset$ and that $\gamma_{i, N}$ is constant on $W$. Let $K \subseteq W$ be a compact subset. There exists $k_{0} \in \mathbb{N}$ such that if $k \geq k_{0}, x, y \in K$ and $d_{G}(x, y)>0$, then

$$
\Phi_{i, N+k|G|}(x) \neq \Phi_{i, N+k|G|}(y) .
$$

Corollary 1.7. If the action of $G$ on $M$ is free, then $\Phi_{i, N}$ is well defined and is an embedding $M / G \hookrightarrow \mathbb{P}\left(H\left(M, A^{\otimes k}\right)^{G^{*}}\right)$ for any $i=1, \ldots, c$ and $N \gg 0$.

Similar statements hold for the asymptotic metric and almost complex properties, in the vein of Theorem 1.1 of [BU2].

\section{Proofs}

Proof of Theorem 1.1. We recall some notation from [BU2, Z, SZ2. Let $A^{*}=$ $A^{-1}$ be the dual line bundle with the induced hermitian stucture $h_{A^{*}}$, and let $A^{*} \supset \mathbb{S} \stackrel{\pi}{\rightarrow} M$ be the unit circle bundle, a strictly pseudoconvex domain. Given the connection, $\mathbb{S}$ has natural riemannian and almost $\mathrm{CR}$ structures. We shall identify functions and half-forms throughout.

As $\mathbb{S}$ is a principal $S^{1}$-bundle, $\mathcal{C}^{\infty}(\mathbb{S})=\bigoplus_{N \in \mathbb{Z}} \mathcal{C}^{\infty}(\mathbb{S})_{N}$, where $\mathcal{C}^{\infty}(\mathbb{S})_{N}$ is the $N$-th isotype for the $S^{1}$-action. We shall identify $\mathcal{C}^{\infty}\left(M, A^{\otimes N}\right)$ and $\mathcal{C}^{\infty}(\mathbb{S})_{N}$ in the standard manner. Set $H(\mathbb{S}):=\bigoplus_{N \in \mathbb{N}} H(\mathbb{S})_{N}$, where $H(\mathbb{S})_{N} \cong H\left(M, A^{\otimes N}\right)$ under this identification; in the integrable projective case, $H(\mathbb{S})$ is the Hardy space of boundary values of holomorphic functions on $A^{*}$. Let $\Pi: L^{2}(\mathbb{S}) \rightarrow H(\mathbb{S})$ be the orthogonal projector and $\tilde{\Pi} \in \mathcal{D}^{\prime}(\mathbb{S} \times \mathbb{S})$ its Schwartz kernel; decompose it as $\tilde{\Pi}=\bigoplus_{N \in \mathbb{N}} \tilde{\Pi}_{N}$, where $\tilde{\Pi}_{N} \in \mathcal{C}^{\infty}(\mathbb{S} \times \mathbb{S})$ is the $N$-th Fourier coefficent. We have $\tilde{\Pi}_{N}(x, y)=\sum_{i=0}^{d_{N}} s_{i}^{N}(x) \otimes \bar{s}_{i}^{N}(y)$, where $\left\{s_{0}^{N}, \ldots, s_{d_{N}}^{N}\right\}$ is an orthonormal basis of $H(\mathbb{S})_{N}$. Let $\tilde{\Phi}_{i, N}: \mathbb{S} \rightarrow H\left(M, A^{\otimes N}\right)^{*}$ be the coherent state map, given by evaluation, which is a lifting of $\Phi_{i, N}$ when the latter is defined. Then $\tilde{\Pi}_{N}(p, q)=$ $\left(\tilde{\Phi}_{i, N}(p), \tilde{\Phi}_{i, N}(q)\right)(p, q \in \mathbb{S})$, where $(\cdot, \cdot)$ denotes the $L^{2}$-hermitian product on $H\left(M, A^{\otimes N}\right)^{*}$.

The induced action of $G$ on $A^{*}$ preserves $\mathbb{S}$ and the riemannian and almost CR structures on $\mathbb{S}$, and the isomorphisms $H(\mathbb{S})_{N} \cong H\left(M, A^{\otimes N}\right)$ are $G$-equivariant. For $N \gg 0$, we have $G$-equivariant decompositions $H(\mathbb{S})_{N}=\bigoplus_{i} H(\mathbb{S})_{i, N}$, where $H(\mathbb{S})_{i, N}$ is the factor consisting of a direct sum of copies of $V_{i}, 1 \leq i \leq c$. Similarly, $H(\mathbb{S})=\bigoplus_{i} H(\mathbb{S})_{i}$. We shall implicitly identify $H(\mathbb{S})_{N}$ and $H(\mathbb{S})_{i, N}$ with $H\left(M, A^{\otimes N}\right)$ and $H\left(M, A^{\otimes N}\right)_{i}$, respectively. For each $i$, let $\Pi_{i}: L^{2}(\mathbb{S}) \rightarrow H(\mathbb{S})_{i}$ denote the orthogonal projection and let $\tilde{\Pi}_{i} \in \mathcal{D}^{\prime}(\mathbb{S} \times \mathbb{S})$ be its Schwartz kernel. For each $i$ and $N$, let $\Pi_{i, N}: L^{2}(\mathbb{S}) \rightarrow H(\mathbb{S})_{i, N}$ be the orthogonal pojection and $\tilde{\Pi}_{i, N}$ its Schwartz kernel, the $N$-th Fourier coefficient of $\tilde{\Pi}_{i}$ : if $\left\{s_{0}^{(i, N)}, \ldots, s_{d_{i, N}}^{(i, N)}\right\}$ is an orthonormal basis of $H(\mathbb{S})_{i, N}$, then

$$
\tilde{\Pi}_{i, N}(p, q)=\sum_{j=0}^{d_{i, N}} s_{j}^{(i, N)}(p) \otimes \overline{s_{j}^{(i, N)}(q)} \quad(p, q \in \mathbb{S}) .
$$

Clearly, $\tilde{\Pi}=\sum_{i=1}^{c} \tilde{\Pi}_{i}$. Notice that the Fourier components of the total and equivariant Szegö kernels, $\Pi_{N}$ and $\Pi_{i, N}$, when restricted to the diagonal in $\mathbb{S} \times \mathbb{S}$, descend to well-defined smooth functions on the diagonal in $M \times M$, that is, with some abuse of language we may write $\Pi_{N}(p, p)=\Pi_{N}(x, x)$ and $\Pi_{i, N}(p, p)=\Pi_{i, N}(x, x)$ for any $p \in \mathbb{S}$ and $x \in M$ with $\pi(p)=x$. This will be done implicitly below. 
By the projection formula, for each $i=1, \ldots, c$ we have

$$
\tilde{\Pi}_{i, N}=\sum_{j=0}^{d_{N}} \Pi_{i}\left(s_{j}^{N}\right) \otimes \bar{s}_{j}^{N}=\left(\operatorname{dim}\left(V_{i}\right) /|G|\right) \cdot \sum_{g} \sum_{j} \bar{\chi}_{i}(g) \rho(g)\left(s_{j}^{N}\right) \otimes \bar{s}_{j}^{N},
$$

where $\rho: G \rightarrow \mathrm{GL}\left(H(\mathbb{S})_{N}\right)$ is the induced representation; explicitly, $\rho(g) \sigma=\sigma \circ g^{-1}$ $\left(g \in G, \sigma \in H(\mathbb{S})_{N}\right)$, where we view $g^{-1}$ as a contactomorphism of $\mathbb{S}$. Thus,

$$
\begin{array}{r}
\tilde{\Pi}_{i, N}(p, q)=\left(\operatorname{dim}\left(V_{i}\right) /|G|\right) \cdot \sum_{g} \sum_{j} \bar{\chi}_{i}(g) s_{j}^{N}\left(g^{-1} p\right) \bar{s}_{j}^{N}(q) \\
=\left(\operatorname{dim}\left(V_{i}\right) /|G|\right) \cdot \sum_{g} \bar{\chi}_{i}(g) \tilde{\Pi}_{N}\left(g^{-1} p, q\right) .
\end{array}
$$

On the diagonal, $\tilde{\Pi}_{i, N}(p, p)=\left(\operatorname{dim}\left(V_{i}\right) /|G|\right) \cdot \sum_{g} \bar{\chi}_{i}(g) \tilde{\Pi}_{N}\left(g^{-1} p, p\right)$. Let $d$ be the geodesic distance function on $M$ and also its pull-back $d \circ \pi$ to $\mathbb{S}$. If $x \in M$ and $G \cdot x \neq\{x\}$, set $a_{x}:=\min \left\{d(g x, x): g \in G \backslash G_{x}\right\}$. Suppose $p \in \mathbb{S}, x=\pi(p)$. Then

$$
\begin{aligned}
\tilde{\pi}_{i, N}(p, p)= & \left(\operatorname{dim}\left(V_{i}\right) /|G|\right) \cdot \sum_{g \in G_{x}} \bar{\chi}_{i}(g) \tilde{\Pi}_{N}\left(g^{-1} p, p\right) \\
& +\left(\operatorname{dim}\left(V_{i}\right) /|G|\right) \cdot \sum_{g \notin G_{x}} \bar{\chi}_{i}(g) \tilde{\Pi}_{N}\left(g^{-1} p, p\right) .
\end{aligned}
$$

By virtue of Lemma 4.5 of [BU2], the latter term is bounded in absolute value by $C \tilde{\Pi}_{N}(p, p) e^{-a_{x}^{2} N / 2}+O\left(N^{(n-1) / 2}\right)$, where $C$ is independent of $x$ and $N$. By (13) of [SZ1] and the definition of dual action, $\tilde{\Pi}_{N}\left(g^{-1} p, p\right)=\alpha_{x}(g)^{N} \tilde{\Pi}_{N}(p, p)$ if $g \in G_{x}$. Thus the former term is

$$
\begin{array}{r}
\left(\operatorname{dim}\left(V_{i}\right) /|G|\right) \cdot\left[\sum_{g \in G_{x}} \bar{\chi}_{i}(g) \alpha_{x}(g)^{N}\right] \tilde{\Pi}_{N}(p, p) \\
=\left(\operatorname{dim}\left(V_{i}\right) /|G|\right) \cdot\left(\alpha_{x}^{N}, \chi_{i}\right)_{G_{x}} \cdot \tilde{\Pi}_{N}(p, p) .
\end{array}
$$

Given the asymptotic expansion for $\tilde{\Pi}_{N}(p, p)$ in BU2 and [Z], $\tilde{\Pi}_{i, N}(p, p) \neq 0$ if $N \gg 0, x \notin B_{i, N}$. This clearly implies the statement.

Proof of Corollary 1.1 Let $V \subseteq M$ be the locus of points with non-trivial stabilizer. By Theorem 8.1 on page 213 of [S] and because the action is effective, $V$ is a union of proper submanifolds of $M$. If $x \in M \backslash V$, then $G_{x}=\{e\}$ and therefore $\gamma_{i, k}(x)=$ $\operatorname{dim}\left(V_{i}\right) \neq 0$ for every $i$ and $N$. By the theorem, there exists $s \in H\left(M, A^{\otimes k}\right)_{i}$ with $s(x) \neq 0$ if $k \gg 0$.

Before coming to the proof of Proposition [1.1 let us dwell on the previous description of the equivariant Szegö kernels $\tilde{\Pi}_{i, k}$ restricted to the diagonal. As is well known, the wave front of the Szegö kernel $\Pi$ is

$$
\left.\Sigma=\left\{\left((p, p),\left(r \alpha_{p},-r \alpha_{p}\right)\right): p \in \mathbb{S}, r>0\right)\right\} \subseteq T^{*}(\mathbb{S} \times \mathbb{S}) \backslash\{0\} .
$$

In the notation of [BdMG], [BU2] we have in fact $\Pi \in J^{1 / 2}(\mathbb{S} \times \mathbb{S}, \Sigma)$. Now we have seen that

$$
\tilde{\Pi}_{i, N}(p, p)=\left(\operatorname{dim}\left(V_{i}\right) /|G|\right) \cdot \sum_{g \in G} \bar{\chi}_{i}(g) \tilde{\Pi}_{N}\left(g^{-1} p, p\right) .
$$

For any $g \in G$ let $\alpha_{g}: \mathbb{S} \times \mathbb{S} \rightarrow \mathbb{S} \times \mathbb{S}$ be the diffeomorphism $(p, q) \mapsto(g p, q)$, and let $\tilde{\Pi}_{g}=\tilde{\Pi} \circ \alpha_{g}^{*} \in \mathcal{D}^{\prime}(\mathbb{S} \times \mathbb{S})$, where $\alpha_{g}^{*}$ denotes pull-back of functions under $\alpha_{g}$. Then $\tilde{\Pi}_{g} \in J^{1 / 2}\left(\mathbb{S} \times \mathbb{S}, \alpha_{g}^{*}(\Sigma)\right)$ and $\tilde{\Pi}_{k}(g p, q)$ is the $k$-th Fourier component of $\tilde{\Pi}_{g}$, 
for every integer $k$. One can then see, arguing as in the proofs of Lemmas 3.5 and 3.6 of [BU2], that $k^{-n} \tilde{\Pi}_{k}(g p, p)$ is bounded in $\mathcal{C}^{1}$ norm, say, for every $g \in G$. The same then holds for $k^{-n} \tilde{\Pi}_{i, k}(x, x)$.

Proof of Proposition 1.1. By the above, in the hypothesis of the proposition for every $x \in M$ there exists $k_{x} \in \mathbb{N}$ such that $x \notin \operatorname{Bs}\left(\left|H\left(M, A^{\otimes k}\right)_{i}\right|\right)$ for every $k \geq k_{x}$. We now make the stronger claim that for every $x \in M$ there exist an open neighbourhood $U_{x}$ of $x$ and $k_{x} \in \mathbb{N}$ such that $U_{x} \cap \operatorname{Bs}\left(\left|H\left(M, A^{\otimes k}\right)_{i}\right|\right)=\emptyset$ for every $k \geq k_{x}$. The statement will follow given the compactness of $M$.

If the claim was false, there would exist $x \in M$ and sequences $k_{j} \in \mathbb{N}$ and $x_{j} \in M(j=1,2, \ldots)$ with $k_{j} \equiv r(\bmod |G|), k_{j} \rightarrow+\infty$ and $x_{j} \rightarrow x$, such that $x_{j} \in \operatorname{Bs}\left(\left|H\left(M, A^{\otimes k_{j}}\right)_{i}\right|\right)$ for every $j$. Thus,

$$
\tilde{\Pi}_{i, k_{j}}\left(x_{j}, x_{j}\right)=0 \quad(j=1,2, \ldots)
$$

while

$$
\tilde{\Pi}_{i, k_{j}}(x, x)=\frac{\operatorname{dim}\left(V_{i}\right)}{|G|} \cdot \gamma_{i, r}(x) \cdot \tilde{\Pi}_{k_{j}}(x, x)+\text { L.O.T. }
$$

where L.O.T. denotes lower order terms in $k_{j}$. Thus, $k_{j}^{-n} \tilde{\Pi}_{i, k_{j}}(x, x)$ is bounded away from zero and therefore the derivatives in $x$ of the sequence of functions $k_{j}^{-n} \tilde{\Pi}_{i, k_{j}}\left(x^{\prime}, x^{\prime}\right)$ are unbounded, a contradiction.

Proof of Corollary 1.2 Let us agree that the irreducible representation corresponding to $i=1$ is just the trivial representation, so that

$$
H\left(M, A^{\otimes N}\right)_{1}=H\left(M, A^{\otimes N}\right)^{G}
$$

for every integer $N$. Then $\bar{\chi}_{1}(g)=1$ for every $g \in G$. Furthermore, for every $x \in M, g \in G_{x}$ and $k \in \mathbb{N}$ we have $\alpha_{x}^{k|G|}(g)=1$. Thus

$$
\gamma_{1, k|G|}(x)=\left|G_{x}\right| \neq 0 \text { for every } x \in M,
$$

and the statement follows from Proposition 1.1 .

Proof of Corollary 1.3. If $M$ is a complex projective manifold and $A$ is ample, we have section multiplication maps

$$
H^{0}\left(M, A^{\otimes \ell}\right)^{G} \otimes H^{0}\left(M, A^{\otimes m}\right)_{i} \longrightarrow H^{0}\left(M, A^{\otimes(\ell+m)}\right)_{i}
$$

for every $i=1, \ldots, c$ and integers $\ell, m$. Thus, for any residue class $0 \leq r \leq|G|-1$ and any sequence of positive integers $k_{i} \gg 0$, by Corollary 1.2 we have a descending chain of base loci:

$$
\begin{array}{r}
\operatorname{Bs}\left(\left|H^{0}\left(M, A^{\otimes r}\right)_{i}\right|\right) \supseteq \operatorname{Bs}\left(\left|H^{0}\left(M, A^{\otimes\left(r+k_{1}|G|\right)}\right)_{i}\right|\right) \\
\supseteq \operatorname{Bs}\left(\left|H^{0}\left(M, A^{\otimes\left(r+\left(k_{1}+k_{2}\right)|G|\right)}\right)_{i}\right|\right) \supseteq \cdots .
\end{array}
$$

This implies the first statement. The rest is obvious.

Proof of Corollary 1.4 If $G_{x}=G$ and $k \equiv r(\bmod |G|)$, then

$$
\tilde{\Pi}_{i, k}(x, x)=\frac{\operatorname{dim}\left(V_{i}\right)}{|G|} \cdot \gamma_{i, r}(x) \cdot \tilde{\Pi}_{k}(p, p) .
$$

Thus, if $\gamma_{i, r}(x)=0$, then $s(x)=0$ for every $s \in H\left(M, A^{\otimes k}\right)_{i}$.

Proof of Corollary 1.5. The first statement follows from Theorem 1.1 while the second is a consequence of the proof of Proposition 1.1 
Proof of Theorem 1.2. Suppose $B_{i, N}=\emptyset$ so that, perhaps after replacing $N$ by $N+k|G|$ for $k \gg 0,\left|H(\mathbb{S})_{i, N}\right|$ is base-point-free. The claim is that if $U^{\prime} \subset U$ is open with compact closure in $U$ and $N \gg 0$, then $\Phi_{i, N}$ is an immersion on $U^{\prime}$. We shall be done by proving that $N^{-1} \Phi_{i, N}^{*}\left(\omega_{\mathrm{FS}}^{(N)}\right)-\omega=O(1 / N)$ on connected compact subsets of $U$, where $\omega_{\mathrm{FS}}^{(N)}$ is the Fubini-Study symplectic form on $\mathbb{P}\left(H\left(M, A^{\otimes k}\right)^{*}\right)$. In turn, this will follow if we prove that $N^{-1} \tilde{\Phi}_{i, N}^{*}\left(\tilde{\omega}_{N}\right)-\pi^{*}(\omega)=O(1 / N)$ on horizontal vectors, over compact subsets of $\mathbb{S}$; here $\tilde{\omega}_{N}=\frac{i}{2} \bar{\partial} \partial \log |\xi|^{2}$ on $H\left(M, A^{\otimes k}\right)^{*} \backslash\{0\}$ (with its natural hermitian structure), and $\pi: \mathbb{S} \rightarrow M$ is the projection.

Now, if $d^{1}$ and $d^{2}$ denote exterior differentiation on the first and second component of $\mathbb{S} \times \mathbb{S}$, respectively, then $N^{-1} \tilde{\Phi}_{i, N}^{*} \tilde{\omega}_{N}=\operatorname{diag}^{*}\left(d^{1} d^{2} \log \tilde{\Pi}_{i, N}\right)$, where $\operatorname{diag}: \mathbb{S} \rightarrow \mathbb{S} \times \mathbb{S}$ is the diagonal map ([SZ2], proof of Theorem 3.1 (b)). If $x, y \in M$ lie in the same connected component $V$ of $U, G_{y}=G_{x}$. Thus $b_{x}:=\left(\alpha_{x}^{N}, \chi_{i}\right)_{G_{x}}$ is constant on $V$, say equal to $b_{V}$. Hence, if $p, q \in \pi^{-1}(V)$ and $x=\pi(p)$,

$$
\tilde{\Pi}_{i, N}(p, q)=\frac{\operatorname{dim}\left(V_{i}\right)}{|G|} \cdot\left\{b_{V} \cdot \tilde{\Pi}_{N}(p, q)+\sum_{g \notin G_{x}} \bar{\chi}_{i}(g) \tilde{\Pi}_{N}(g p, q)\right\} .
$$

By the proof of Theorem 3.1 (b) of [SZ2], $(i / 2 N) \operatorname{diag}^{*}\left(d^{1} d^{2} \log \Pi_{N}\right) \rightarrow \pi^{*} \omega$ in $\mathcal{C}^{k}$-norm for any $k$ on $M$. Therefore, we shall be done by proving that

$$
N^{-1} d_{1} d_{2}\left(\tilde{\Pi}_{N}(g p, q) / \tilde{\Pi}_{N}(p, q)\right) \rightarrow 0
$$

and

$$
N^{-1} d_{1}\left(\tilde{\Pi}_{N}(g p, q) / \tilde{\Pi}_{N}(p, q)\right) \wedge d_{2}\left(\tilde{\Pi}_{N}\left(g^{\prime} p, q\right) / \tilde{\Pi}_{N}(p, q)\right) \rightarrow 0
$$

for $g, g^{\prime} \notin G_{x}$ near compact subsets of $\operatorname{diag}(V)$.

Then let $K \subset V$ be a compact subset, and suppose $x \in K, g \notin G_{x}$, and $u, v \in T_{x} M$ have unit length. Let $U, V$ be horizontal vector fields of unit length on $\mathbb{S}$, of unit length near $\mathbb{S}_{x}$ and extending the horizontal lifts of $u$ and $v$. We want to estimate $N^{-1} U_{1} \circ V_{2}\left(\tilde{\Pi}_{N}(g p, q) / \tilde{\Pi}_{N}(p, q)\right)$ over $K$, where $U_{1}=(U, 0)$ and $V_{2}=(0, V)$ are horizontal vector fields on $\mathbb{S} \times \mathbb{S}$.

Let us consider again the distribution $\tilde{\Pi}_{g}=\alpha_{g}^{*} \tilde{\Pi} \in J^{1 / 2}\left(\mathbb{S} \times \mathbb{S}, g^{*} \Sigma\right)$, discussed before the proof of Proposition 1.1. If $P$ is a horizontal differential operator of degree $\ell$ on $\mathbb{S} \times \mathbb{S}$, its principal symbol vanishes on $g^{*} \Sigma$ and therefore $P\left(\tilde{\Pi}_{g}\right) \in$ $J^{(\ell+1) / 2}\left(\mathbb{S} \times \mathbb{S}, \alpha_{g}^{*} \Sigma\right)$. As in [BU2], Lemma 4.5 , for $k \in \mathbb{N}$ we can find $\nu_{g, P, k} \in$ $\mathcal{C}^{\infty}(\mathbb{S})$, having an asymptotic expansion $\nu_{g, P, k}(p)=\sum_{j=0}^{\infty} k^{n+(\ell-j) / 2} f_{g, P, k}^{(j)}(p)$, and real phase functions $\alpha_{g, P, k} \in \mathcal{C}^{\infty}(\mathbb{S} \times \mathbb{S})$ such that

$$
G(p, q)=\sum_{k} \nu_{g, P, k}(p) e^{i \alpha_{g, P, k}(p, q)} e^{-k d(g p, q)^{2} / 2} \in J^{(\ell+1) / 2}\left(\mathbb{S} \times \mathbb{S}, \alpha_{g}^{*} \Sigma\right)
$$

and $P\left(\tilde{\Pi}_{g}\right)-G \in J^{\ell / 2}\left(\mathbb{S} \times \mathbb{S}, \alpha_{g}^{*} \Sigma\right)$. Since $P\left(\tilde{\Pi}_{g}\right)$ has definite (even) parity, we may assume without loss of generality that so does $G$. Therefore, the above asymptotic expansions may be assumed to go down by integer steps: $\nu_{g, P, k}(p)=$ $\sum_{j=0}^{\infty} k^{n+\ell / 2-j} f_{g, P, k}^{(j)}(p)$, and

$$
\left|P\left(\tilde{\Pi}_{N}(g p, q)\right)\right|=\nu_{g, P, 0}(p) \cdot e^{-N d(g p, q)^{2} / 2}+O\left(N^{n+\ell / 2-1}\right) .
$$

Because $K \subset U$ is compact and $g \notin G_{x}$ for $x \in K$, there is $\epsilon>0$ such that $d(g p, p)>\epsilon$ for all $p \in \pi^{-1}(K)$. Thus, $P\left(\tilde{\Pi}_{N}^{(g)}\right)(p, p)=O\left(N^{m+(\ell-1) / 2}\right)$ 
on $\pi^{-1}(K)$. Developing $N^{-1} U_{1} \circ V_{2}\left(\tilde{\Pi}_{N}(g p, q) / \tilde{\Pi}_{N}(p, q)\right)$, we see that $N^{-1} U_{1} \circ$ $V_{2}\left(\tilde{\Pi}_{N}(g p, q) / \tilde{\Pi}_{N}(p, q)\right)=O(1 / N)$ over $K$, uniformly in $U$ and $V$. This proves (2); the proof of the other estimate is similar.

Proof of Proposition 1.2. Notation being as above, we may assume that $K$ is $G$ invariant. Suppose then, by contradiction, that for a sequence $k_{j} \rightarrow+\infty$ we can find $x_{k_{j}}, y_{k_{j}} \in K$ with $d_{G}\left(x_{k_{j}}, y_{k_{j}}\right)>0$ and $\Phi_{i, N+k_{j}|G|}\left(x_{k_{j}}\right)=\Phi_{i, N+k_{j}|G|}\left(y_{k_{j}}\right)$. Set $N_{j}=N+k_{j}|G|$.

I claim that $d_{G}\left(x_{k_{j}}, y_{k_{j}}\right) \leq C / \sqrt{N_{j}}$. Following [BU2], proof of Corollary 4.6, pick $p_{k_{j}} \in \pi^{-1}\left(x_{k_{j}}\right), q_{k_{j}} \in \pi^{-1}\left(y_{k_{j}}\right)$. Then $\tilde{\Phi}_{i, N_{j}}\left(x_{k_{j}}\right)=\lambda_{j} \tilde{\Phi}_{i, N_{j}}\left(y_{k_{j}}\right)$ for some $\lambda_{j} \in$ $\mathbb{C}$; it follows that $\left\|\tilde{\Phi}_{i, N_{j}}\left(p_{k_{j}}\right)\right\|^{2}=\left|\lambda_{j}\right|^{2} \cdot\left\|\tilde{\Phi}_{i, N_{j}}\left(q_{k_{j}}\right)\right\|^{2}$. However, $\left\|\tilde{\Phi}_{i, N_{j}}(p)\right\|^{2}=$ $\tilde{\Pi}_{i, N_{j}}(p, p)(p \in \mathbb{S})$, and therefore by (1) above $\left|\lambda_{j}\right|=1+O\left(N_{j}^{-1 / 2}\right)$. We also have $\left|\lambda_{j}\right| \tilde{\Pi}_{i, N_{j}}\left(p_{k_{j}}, p_{k_{j}}\right)=\left|\tilde{\Pi}_{i, N_{j}}\left(p_{k_{j}}, q_{k_{j}}\right)\right|$, and on the other hand, again by (1),

$$
\left|\tilde{\Pi}_{i, N_{j}}\left(p_{k_{j}}, q_{k_{j}}\right)\right| \leq C\left|\tilde{\Pi}_{i, N_{j}}\left(p_{k_{j}}, p_{k_{j}}\right)\right| e^{-N_{j} d_{G}(p, q)^{2} / 2}+O\left(k_{j}^{n-1 / 2}\right) .
$$

We conclude that $d_{G}\left(p_{k_{j}}, q_{k_{j}}\right) \leq C / \sqrt{k_{j}}$, as claimed. Hence, after replacing $x_{k_{j}}$ by $g_{j} \cdot x_{k_{j}}$ for a suitable $g_{j} \in G$, we may assume $d\left(x_{k_{j}}, y_{k_{j}}\right) \leq C / \sqrt{N_{j}}$ and $d\left(x_{k_{j}}, y_{k_{j}}\right)=$ $d_{G}\left(x_{k_{j}}, y_{k_{j}}\right)$ for every $j$.

Since $d(g x, x)>\epsilon$ for some fixed $\epsilon>0$ and all $x \in K$ and $g \notin G_{x}, x_{k_{j}}$ is the only point in $G \cdot x_{k_{j}}$ minimizing the distance from $y_{k_{j}}$, for every $j$.

We may now apply the argument of the proof of Theorem 3.2 (b) of [SZ2], with minor changes.

\section{REFERENCES}

[BU1] D. Borthwick, A. Uribe, Almost complex structures and geometric quantization, Math. Res. Lett. 3 (1996), 845-861. MR 98e:58084

[BU2] D. Borthwick, A. Uribe, Nearly Kählerian embeddings of symplectic manifolds, Asian J. Math. 4 (2000), 599-620. MR 2001m:53166

[BdM] L. Boutet de Monvel, Hypoelliptic operators with double characteristics and related pseudodifferential operators, Comm. Pure Appl. Math. 27 (1974), 585-639. MR 51:6498

[BdMG] L. Boutet de Monvel, V. Guillemin, The spectral theory of Toeplitz operators, Ann. Math. Studies 99, Princeton University Press (1981). MR 85j:58141

[GU] V. Guillemin, A. Uribe, The Laplace operators on the nth tensor powers of a line-bundle, Asympt. Anal. 1 (1988), 105-113. MR 90a:58180

[P] R. Paoletti, The asymptotic growth of equivariant sections of positive and big line bundles, preprint

[SZ1] B. Shiffman, S. Zelditch, Universality and scaling of correlations between zeros on complex manifolds, Inv. Math. 142 (2000), 351-395. MR 2002f:32037.

[SZ2] B. Shiffman, S. Zelditch, Asymptotics of almost holomorphic sections of ample line bundles on symplectic manifolds, J. Reine Angew. Math. 544 (2002), 181-222. MR 2002m:58043

[S] S. Sternberg, Lectures on differential geometry, Prentice Hall, Englewood Cliffs, N.J. (1964). MR 33:1797

[T] G. Tian, On a set of polarized Kähler metrics on algebraic manifolds, J. Diff. Geom. 32 (1990), 99-130. MR 91j:32031

[Z] S. Zelditch, Szegö kernels and a theorem of Tian, Int. Math. Res. Notices 6 (1998), 317-331. MR 99g:32055

Dipartimento di Matematica e Applicazioni, Universitá di Milano Bicocca, Via BicocCa Degli Arcimboldi 8, 20126 Milano, Italy

E-mail address: roberto.paoletti@unimib.it 